\title{
Regular physical exercise: evidence for health benefits in OSA patients?
}

\author{
Wolfgang Schobersberger
}

Received: 11 January 2013 / Accepted: 15 January 2013 / Published online: 25 January 2013

(C) Springer-Verlag Berlin Heidelberg 2013

Obstructive sleep apnea syndrome (OSA) is recognized as a growing public health problem with an increasing prevalence up to $5 \%$ in men. As an independent risk factor, it is associated with several diseases such as hypertension, coronary heart disease, or stroke thus increasing cardiovascular morbidity and mortality [1]. Furthermore, classical OSA symptoms are daytime sleepiness, tiredness, fatigue, neurocognitive dysfunction, and depression, worsening the individual's quality of life. Physical exercise in general has impressively been shown to be an essential factor in preventive medicine. For example, regular physical training (aerobic training and/or resistance training) improves respiratory function in patients with chronic obstructive pulmonary disease [2] and improves all risk factors of patients suffering from metabolic syndrome [3]. Even in the primary prevention of some cancers (e.g., mamma carcinoma and colon carcinoma), physical exercise plays a crucial role [4]. Therefore, regular physical training is suggested to be effective in several diseases such as a medication and 10,000 steps per day were recommended as preventive measure [5]. Is there a causal link between OSA severity and lack of physical exercise? The approach to answer this question is dual. First, we can check prospective studies on the effects of regular physical training on OSA symptoms, and second, we can focus on possible associations between training and OSA symptoms in a retrospective way. In the last months, interesting studies were published, showing that exercise training is associated with a reduced incidence of mild and moderate sleep-disordered breathing [6] and that physical

W. Schobersberger $(\bowtie)$

Institute for Sports Medicine,

Alpine Medicine and Health Tourism,

Private University for Health Sciences, Medical Informatics

and Technology (UMIT), Eduard Wallnöfer Zentrum 1,

6060 Hall, Tyrol, Austria

e-mail: wolfgang.schobersberger@uki.at exercise improves selected aspects of daytime functioning in OSA patients [7].

In this issue of S\&B, Verwimp et al. [8] report on data of their retrospective study with moderate to severe OSA patients regarding possible associations between sleep parameters and quality of life on one hand and daily physical activity on the other hand. Up to now, only preliminary results were published assuming that there is an inverse relationship between physical activity and OSA severity. The study of Verwimp et al. could add new information on this topic by a real detection of the individual activity and energy expenditure with an established technology of Bodymedia SenseWear Pro Armband activity monitor over a 5-day period. Regarding physical exercise, the authors reported significant inverse correlations between the steps walked and the presence of comorbidities (hypertension, diabetes, obesity) and between the apnea hypopnea index in REM sleep. Moreover, the patients linked their subjective reduction of quality of life with their measured reduced physical activity. The authors concluded that the low level of physical activity is associated with OSA severity in somnolent moderate to severe OSA patients. Therefore, one could speculate that regular physical activity and training may reduce not only OSA-associated comorbidities but improve health-related quality of life. Randomized controlled trials ("exercise studies") are required in order to get detailed information on the optimal effects of physical exercise in OSA patients.

Conflict of interest None

\section{References}

1. Martin JM, Carizzo SJ, Vicente E, Agusti AG (2005) Long-term cardiovascular outcomes in men with obstructive sleep apnoehypopnoea with or without treatment with continuous positive airway pressure: an observational study. Lancet 365:1046-1043 
2. Strasser B, Siebert U, Schobersberger W (2012) Effects of resistance training on respiratory function in patients with chronic obstructive pulmonary disease: a systematic review and meta-analysis. Sleep Breath. doi:10.1007/s11325-012-0676-4

3. Strasser B, Siebert U, Schobersberger W (2010) Resistance training in the treatment of the metabolic syndrome: a systematic review and meta-analysis of the effect of resistance training on metabolic clustering in patients with abnormal glucose metabolism. Sports Med 40:397-415

4. Wiggins MS, Simonavice EM (2010) Cancer prevention, aerobic capacity, and physical functioning in survivors related to physical activity: a recent review. Cancer Manag Res 2:157-164
5. Tudor-Locke C, Basset DR Jr (2004) How many steps/day are enough? Preliminary pedometer indices for public health. Sports Med 34:1-8

6. Awad KM, Malhotra A, Barnet JH, Quan SF, Peppard PE (2012) Exercise is associated with a reduced incidence of sleep-disordered breathing. Am J Med 125:485-490

7. Kline CE, Ewing GB, Burch JB, Blair SN, Durstine JL, Davis JM, Youngstedt SD (2012) Exercise training improves selected aspects of daytime functioning in adults with obstructive sleep apnea. J Clin Sleep Med 8:357-365

8. Verwimp J, Amaye L, Bruyneel M (2013) Correlation between sleep parameters, physical activity and quality of life in somnolent moderate to severe obstructive sleep apnea adult patients. Sleep Breath (in press) 